\title{
Análise emocional de produtos de design baseada em expressão facial
}

\author{
Emotional analysis of design products based on facial expression
}

Cláudio Santos de Almeida

Design emocional, reconhecimento facial, expressões emocionais, método de análise

\begin{abstract}
Este artigo trata da descrição de um método de análise de produtos baseado na coleta de informações sobre o estado emocional de sujeitos através de reconhecimento de emoções baseado em modificações da face. Fundamentado na classificação de Donald Norman sobre os níveis de interação dos objetos de design com o ser humano, o artigo apresenta um modelo de análise que pode ser usado para obter informações sobre o nível reativo de design. Propõe o uso de Facial Action Coding System através de técnicas automatizadas de reconhecimento facial de forma a reduzir a subjetividade e garantir a praticidade de uso em aplicações dentro e fora de ambientes controlados. Conclui indicando os passos necessários ao desenvolvimento do método a ser aplicado em análise de produtos no mercado brasileiro e iniciando uma discussão acerca dos aspectos culturais do conceito de satisfação no uso de um produto de design.
\end{abstract}

Emotional design, facial recognition, emotional expressions, techniques, analysis methods

This paper presents the description of an analysis method for design products based on facial expressions. Using the Donald Norman's emotional design levels classification, the paper presents an analysis model that can be used to gain information from the reactive level. It proposes the use of Facial Action Coding System (FACS) and automated techniques from Computer Vision and Affective Computing to improve the accuracy and efficiency of the system in controlled and non-controlled environments. It concludes with a description of the steps for the development of the method to be applied to products in the Brazilian market and initiating a discussion on cultural aspects of the concept of satisfaction applied to the use of design products.

\section{Introdução}

A análise emocional do design tornou-se mais conhecida no Brasil através do livro Design Emocional (Emotional Design) (Norman, 2004), que trata da observação, através de artigos científicos publicados em diversas partes do mundo, da influência decisiva de aspectos emocionais na usabilidade de objetos de design. Baseado nessas observações, Norman propôs uma classificação das relações de design com usuários de produtos em três níveis: o nível reflexivo, o nível comportamental e o nível reativo.

O nível reflexivo do design é aquele no qual a relação com o objeto é essencialmente intelectiva. Nesse nível, a análise racional é preponderante. Norman utiliza como exemplo, objetos controversos, de nenhuma usabilidade, como chaleiras com bicos invertidos, ou miniaturas de monumentos, que não tem necessariamente utilidade funcional ou estética, mas que são apelos ao ato de representar um lugar, um momento ou um contexto histórico. São relações que só fazem sentido através de análises comparativas.

O nível comportamental é o nível da usabilidade. Da relação prática do usuário com o uso definido do objeto. Ao usar um objeto, as características percebidas pelo usuário nesse nível referem-se a sua utilidade e praticidade de uso. A facilidade de uso, a ergonomia e a eficácia de um objeto de design situam-se nesse nível.

O nível reativo é o da emoção não racionalizada. É a ação do gostar subjetivo sem a mediação do parecer cognitivo (figurativamente). Norman usa o cinema para representar esse nível comparativamente com o nível reflexivo: quando vamos ao cinema assistir a um filme, e passamos a observar esteticamente a cena, o figurino ou a iluminação estamos analisando o filme. Mas quando imergimos emocionalmente na trama do filme, todos esses componentes contribuem para 
uma experiência integrada, cujo apelo é essencialmente emocional e que nos leva a participar do filme. É a esse tipo de resposta emocional que Norman qualifica como sendo do nível Reativo.

\section{A relação do usuário com o produto em diferentes níveis}

Cada um dos níveis é observado pelo usuário, consciente ou inconscientemente quando realiza uma ação de compra ou de uso. Nenhuma relação de design ocorre apenas em um dos níveis. Ela acontece simultaneamente em todos os três níveis e de forma integrada. Entretanto, essa relação pode ocorrer em maior ou menor grau em cada um dos níveis de acordo com as circunstâncias de uso, com o estado emocional do usuário ou com o contexto.

\section{A construção do objeto de design e os instrumentos de análise de design}

Existem métodos diferentes para analisar cada um dos níveis. Os métodos derivados de análises dialéticas, estéticas ou históricas tendem a resolver problemas e encontrar perspectivas para o nível reflexivo. Os diversos métodos de $\mathrm{HCl}$, usabilidade e ergonomia focam teoricamente no âmbito comportamental do design. Os métodos provindos da análise psicológica das emoções, e das teorias do comportamento são essencialmente dirigidos ao estudo das relações no nível reativo do design.

Cada método é acompanhado de diferentes tipos de instrumentos de coleta. No nível reativo, pesquisadores utilizam métodos provenientes do estudo psicológico das emoções de forma a obter informações sobre o estado emocional do sujeito de forma não verbal. Um destes métodos provém dos estudos da expressão facial das emoções.

\section{Análise de emoções baseada na expressão facial}

A face humana é uma fonte de informação dos estados emocionais do ser humano (Ekman, 1993; Russel, 1994; Cohn et.al., 1999; Tracy et. al., 2009; Popa et. al. 2010). Um dos primeiros estudos sistemáticos sobre a expressão emocional na face do ser humano remonta a Darwin. Um dos dados obtidos de seus estudos e que é fundamento de subsídios para a análise facial é que analisar estados emocionais em seres humanos pode levar a subjetividades porque a simples observação de estados emocionais por observadores pode provocar estados emocionais involuntários no próprio observador, e assim, a análise e julgamento dos estados emocionais poderia ser comprometido (Darwin, 1896).

Devido a essa subjetividade, pesquisadores desenvolveram vários sistemas de identificação emocional através da face cujo objetivo é manter consistência nos dados coletados e analisados em diferentes circunstâncias e por diferentes pesquisadores. (Ekman, 1969; Ekman \& Frasier, 1978; Izard, 1971; Izard, 1979). Um dos aspectos relevantes da análise facial é que ela é pouco invasiva, se comparada a estudos de respostas emocionais com eletrodos e outros tipos de captura de informação fisiológica. Essa característica associada a facilidades de uso discutidas adiante favorecem ao uso de um método de análise facial aplicado ao estudo de relações de design no nível reativo.

\section{Desenvolvimento}

\section{Validade de um sistema de análise facial para a análise emocional de produtos}

A consistência de um sistema de análise emocional baseado no reconhecimento facial, independente do seu uso, depende de dois fatores: o grau de subjetividade e a praticidade na aplicação.

Um dos mais bem sucedidos casos de resolução de subjetividade na análise é o método denominado Facial Action Coding System (FACS), que consiste em um sistema que classifica modificações da anatomia da face humana através de um conjunto pré-definido de movimentos musculares. Cada movimento distinto é catalogado na forma de uma Unidade de Ação, ou Action Unit, em inglês, que estabelece a posição muscular da face de acordo com um banco de imagem padrão (Ekman \& Frasier, 1978). Ekman comprovou, através de estudos sistemáticos em diversas culturas em diferentes continentes, que certos estados emocionais provocam modificações musculares faciais semelhantes, em particular para as expressões da raiva, nojo, tristeza, alegria e surpresa, denominadas no método emoções básicas (Ekman, Sorenso \& Friesen, 1969). FACS não realiza uma análise do estado emocional envolvida no movimento relacionado com a emoção 
diretamente. Essa análise é feita posteriormente, e não necessariamente pelo mesmo grupo de pesquisadores através de outro método denominado EMFACS (Friesen \& Ekman, 1984). Por conta dessa abordagem, FACS é considerado o mais consistente método de análise emocional baseado em expressões faciais (Cohn, Zlochower, Lien \& Kanade, 1999).

Um dos pressupostos de FACS é a necessidade de comparar as expressões faciais capturadas com as imagens de um banco estável de imagens previamente identificado como sendo de determinada emoção por um grupo de sujeitos. Quanto maior o grupo de sujeitos e mais variado, mais credibilidade o banco de imagens tem para servir como instrumento de comparação com as expressões obtidas.

Ekman desenvolveu as imagens de modificação facial, ou pictures of facial affect (POFA) (Ekman, 1993), que são um banco de imagens de faces humanas que obtém um alto índice de reconhecimento da sua sugestão emocional em diferentes culturas. As imagens podem ser capturadas através de fotos (imagens estáticas) ou filmes (imagens em movimento). Em ambos os casos é interessante usar uma taxa de captura superior a 60 quadros por segundo, o que assegura a captura de modificações musculares que ocorram em um intervalo de tempo muito curto.

Vários bancos de imagens foram desenvolvidos, utilizando diferentes grupos étnicos e posições faciais. Um dos mais conhecidos e utilizados bancos de imagens de reconhecimento de expressões faciais é o banco on-line Cohn-Kanade (Wang \& Yin, 2006).

Outros conjuntos baseados no mesmo princípio foram desenvolvidos para contemplarem outras emoções. Tracy, Robins e Schriber desenvolveram um conjunto de imagens reconhecíveis para analisar três emoções auto-conscientes além das seis emoções básicas: a vergonha, o orgulho e o constrangimento (Tracy et.al., 2009). Entretanto, tais bancos não tem ainda a mesma validade que os bancos mais estáveis POFA e Cohn-Kanade.

$\mathrm{O}$ outro aspecto importante diz respeito à praticidade de uso. Foram identificados mais de 7000 diferentes tipos de combinações de AUs e pesquisas abrangentes podem tomar até 10 horas de codificação por minuto de comportamento observado por indivíduo pesquisado (Ekman, 1982). Uma pesquisa com um número não superior a 10 indivíduos pode, dependendo do tempo de análise de cada indivíduo, exigir a participação de dezenas de pesquisadores durante muitos meses. Essa limitação mostra-se particularmente importante, se considerarmos que, para tornarse proficiente em FACS um pesquisador precisa de pelo menos 100 horas de treinamento (Ekman, 1982; Friesen \& Ekman, 1984; Cohn, Zlochower, Lien \& Kanade, 1999) e que, além disso, o treinamento pode esvanecer no tempo (Bakeman \& Gottman, 1986; Martin \& Bateson, 1986).

Como a subjetividade do método de análise facial é um problema resolvido em um grau aceitável, resta encontrar soluções para a sua aplicabilidade.

\section{Soluções para a aplicabilidade}

Para servir de instrumento de coleta e análise de dados para análise emocional em produtos de design, a técnica de coleta deve ser capaz de observar o usuário em situações fora de ambiente laboratorial e deve ser capaz de sintetizar dados de forma objetiva. Os dados devem ser analisados de forma a obter-se a informação desejada, que no caso consiste no estado emocional do indivíduo num dado instante de sua experimentação como usuário do produto de design ou ao longo de uma trajetória de tempo de forma a estabelecer uma média ou dados quantitativos no tempo que estabelecem a variabilidade emocional.

Pelas limitações citadas acima, FACS e EMFACS não são viáveis como instrumentos de coleta e análise de dados de emoções baseados em respostas faciais para a análise emocional de produtos de design, se aplicados diretamente.

Existem estudos para o desenvolvimento de sistemas mais ágeis e práticos de análise emocional baseados em expressões faciais. Ekman tentou desenvolver um sistema mais adaptável e simples à análise emocional baseado em expressões faciais denominado MicroExpression Training Tool (METT) e diversos pesquisadores estudam a possibilidade de automatizar o reconhecimento facial através de sistemas computadorizados (Cohn et. al., 1999; Wang \& Yin, 2006; Popa et. al., 2010).

Micro Expression Training Tool é uma técnica de custo mais baixo que FACS em capacitação do pesquisador e na avaliação dos dados. Sua premissa é de tentar fazer o observador identificar as emoções nos sujeitos observados sem, contudo identificar-se com elas. Exige uma capacitação 
mais simples e pode ser aplicado diretamente nos sujeitos. Realizamos um estudo preliminar com três sujeitos para analisar a possibilidade de uso de METT em interfaces gráficas de jogos (Almeida, 2009). O resultado, entretanto, é subjetivo. Por conta da subjetividade, um grande número de pesquisas semelhantes precisa ser realizado para poder garantir que um determinado resultado emocional é válido e consistente. Como, entretanto, nenhum caso é igual, pois o observador é diferente e o observado também, a subjetividade pode persistir após extensas investigações e não há registro do resultado. Apenas registro das ações. Dados podem ser cruzados de forma a aumentar a objetividade, mas nesse caso, o problema da praticidade torna-se relevante.

Os sistemas automatizados exigem algoritmos de reconhecimento facial. Basicamente, um algoritmo de reconhecimento facial é uma rotina de passos que permite a um sistema computadorizado reconhecer partes do rosto e modificações da face. O reconhecimento facial é uma parte da visão computacional. Existem basicamente dois tipos de sistemas de reconhecimento facial. Aqueles que se baseiam em imagens estáticas e os que usam imagens em movimento (Wang \& Yin, 2006). Um sistema de captura de posicionamento facial precisa, em primeiro lugar, reconhecer o padrão do rosto. Existem câmeras fotográficas e filmadoras que localizam automaticamente a posição da boca, dos olhos e modificações como sorrisos. São exemplos de reconhecimento facial e de posicionamento facial. Para que o sistema possa analisar emoções, entretanto, um segundo passo é necessário. Torna-se necessário que o sistema associe determinada posição ou variação facial a uma determinada emoção. Como dito anteriormente, as expressões faciais são associadas a emoções de forma subjetiva. Seis emoções foram reconhecidas como universais por Ekman após estudo em diferentes culturas em diferentes partes do mundo. Entretanto, para assegurar que a expressão facial de uma emoção seja reconhecida pelo sistema computadorizado da forma como seria reconhecida por uma média de indivíduos, é necessário que haja uma base comparativa. Os sistemas computadorizados também necessitam de uma base estável de fotos. Cohn, Zlochower, Lien e Kanade desenvolveram um sistema de análise emocional da face através de pontos de marcação (featured points) utilizando o sistema de codificação de FACS e validando o sistema através de uma verificação cruzada com pesquisadores certificados em FACS (Cohn et. al., 1999). Wang e Yin desenvolveram um sistema de modelagem topográfica estática para reconhecimento de expressões faciais em fotografias e utilizaram MMI e Cohn-Kanade como bases fotográficas (Wang \& Yin, 2007). Mais recentemente Popa, Rothkrantz e Wiggers desenvolveram um sistema de apreciação de produtos usando reconhecimento automático de análise de expressões faciais usando a base de imagens CohnKanade (Popa et. al., 2010).

Destes sistemas, o de Popa é o que mais diretamente pretende ter utilidade em ambientes não controlados. Popa utiliza um modelo de reconhecimento facial denominado Active Appearance Model para delimitar áreas chave do rosto. Dessas áreas dados são extraídos através do método denominado Optical Flow Estimation. Por fim, os dados são analisados na forma de expressões emocionais através de modelos Hidden Markow.

Independente dos modelos utilizados, a forma do método de Popa é relevante para o desenvolvimento de um modelo de análise emocional baseado na face porque contêm os três estágios pelos quais um método automatizado de expressões emocionais pode ser desenvolvido. Em primeiro lugar, identificar a face, por partes desta. Em segundo, analisar (no caso) modificações da posição. Em terceiro, relacionar essas modificações com expressões emocionais.

Popa também utiliza Cohn-Kanade, que é um banco de imagens de expressões emocionais padrão, o que implica em ser um sistema baseado em FACS, já que Cohn-Kanade é um banco de expressões faciais baseado nas modificações musculares definidas por FACS. A proposição do método que faremos a seguir é similar a esta, com algumas modificações fundamentais.

\section{Método de análise emocional de produtos baseados em expressões faciais para casos brasileiros.}

No Brasil o design alcançou, a mais de quatro décadas, padrão internacional de consumo. Produtos de design gráfico e industrial servem de modelo para produtos em todo o mundo, desde soluções de mobiliário até produções gráficas e digitais. É importante que os métodos de análise sejam enriquecidos na mesma base da importância do design brasileiro. Aos métodos existentes para análise de produtos, propomos agregar o método que descrevemos adiante e cujo procedimento de desenvolvimento é o assunto da conclusão deste artigo.

Para que o método seja considerado válido dentro dos padrões de FACS é necessário que um 
banco de imagens padrão seja utilizado como forma de aferição do grau de precisão. É um objetivo possível e desejável que os acertos do sistema sejam de acima de $90 \%$ na comparação com a média dos resultados obtidos por pesquisadores habilitados em FACS. O método deve conter a seguinte configuração mínima: uma câmera filmadora para a captura de imagens em movimento, um computador para armazenar as imagens filmadas durante o experimento, um método de reconhecimento facial, um método de diferenciação da modificação da posição facial, um método de reconhecimento das emoções e uma interface de resultado apresentada ao pesquisador, que deve ser ergonomicamente apropriada ao pesquisador em design. Podemos observar um sistema que atende parcialmente a essas definições em

http://www.youtube.com/watch?v=n8wJ8tjmnmU (último acesso em 13 de fevereiro de 2011). No sistema apresentado, as emoções básicas são classificadas do lado direito da tela instantaneamente (Figura1).

Figura 1: eMotion. Um sistema de classificação automático de emoções. Do lado direito da tela, as seis emoções básicas e mais o estado neutro são apresentadas em variações de 0 a 100\%.

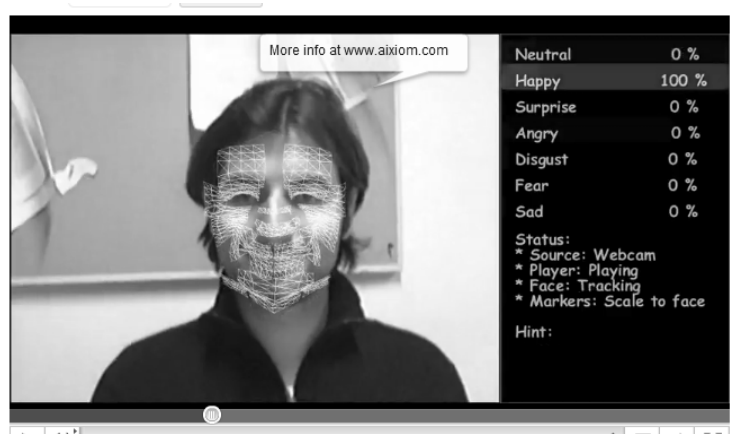

Há motivos que indicam a necessidade de usar-se um banco de imagens brasileiro ao invés dos tradicionais Cohn-Kanade ou POFA. Um dos aspectos mais importantes diz respeito aos caracteres gênicos brasileiros e talvez a sua forma básica de expressão emocional. Como bancos de imagem não foram desenvolvidos com brasileiros e os brasileiros não tem a mesma matriz gênica que os vizinhos latinos ou que outros países do mundo, em virtude de seu alto grau de miscigenação e da quantidade de gerações em que o processo de miscigenação vem ocorrendo, sendo o único país em que isso ocorre em escala nacional e com um grupo representativo de indivíduos, é prudente desenvolver e validar um banco de expressões emocionais padrão (standardized emotion expressions). Tracy, Robins e Schriber descrevem os passos necessários ao desenvolvimento de um banco deste tipo. O banco deve ser fotografado em ângulos precisos e os modelos devem ser orientados a realizarem expressões emocionais voluntárias. Após esse preceito as imagens das expressões devem ser validadas por pesquisadores habilitados no reconhecimento de emoções (Tracy et at., 2009). Quanto mais indivíduos estabelecerem a média das validações, mais acurado e validado torna-se o banco. Mesmo que os indivíduos que visualizam o banco de imagens não sejam proficientes em FACS, estes podem aumentar o grau de credibilidade do banco de imagens se o número de indivíduos for significativo.

Por fim, deve-se levar em conta um aspecto importante da relação entre emoção sentida e a análise reflexiva da satisfação pessoal. A sistematização do método, os passos necessários a serem realizados e comentários acerca da satisfação são discutidos adiante, na conclusão deste artigo.

\section{Conclusão}

A figura 2 define a sistematização de um método de análise emocional por reconhecimento automatizado da face. 
Figura 2.

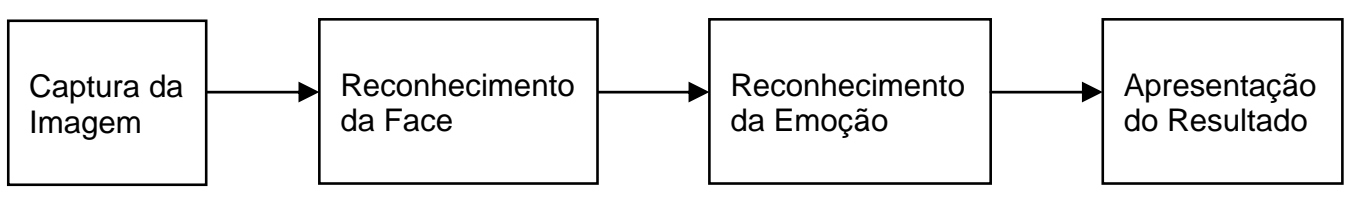

O sistema acima permite registrar as emoções instantâneas em uma experimentação, quer seja na aquisição/observação de um produto, quer seja no seu uso. Entretanto, é provável que a satisfação de nível reativo a que se refere Norman não seja observada no nível reflexivo como tal apenas através ou como substrato de uma única impressão emocional, mas sim por um conjunto delas. Em outras palavras, é possível que a satisfação de obtenção e uso de um produto não seja diretamente associado a uma única emoção, mas a um conjunto delas (Almeida, 2009).

Para registrar as emoções no tempo, é preciso que o sistema guarde mais um conjunto de informações, que é a linha de variação emocional em cada uma das seis emoções básicas e mais o estado neutro. Podemos exemplificar isso na forma de um gráfico hipotético da variação emocional ao longo do tempo de uma sequência linear de capturas instantâneas de uma determinada emoção (Figura 3).

Figura 3. Gráfico de variação emocional obtido através de momentos de captura.

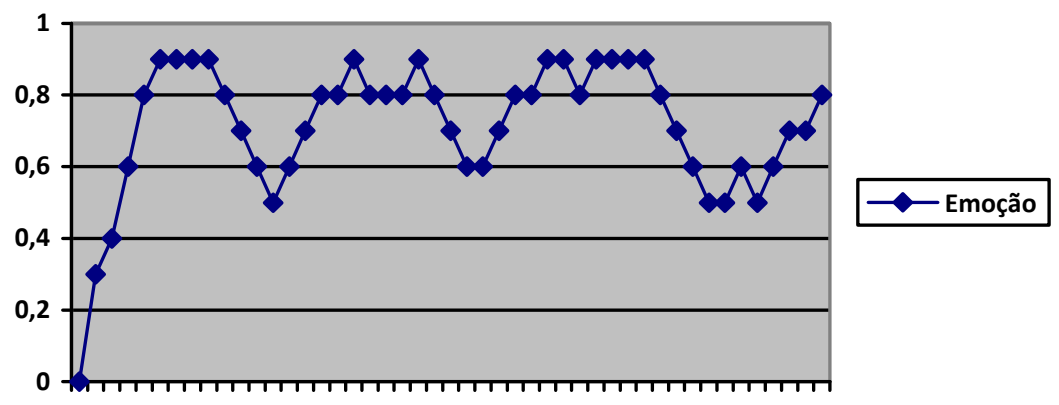

\section{Sistema proposto.}

A figura 3 define o sistema proposto para o caso brasileiro.

Figura 3.

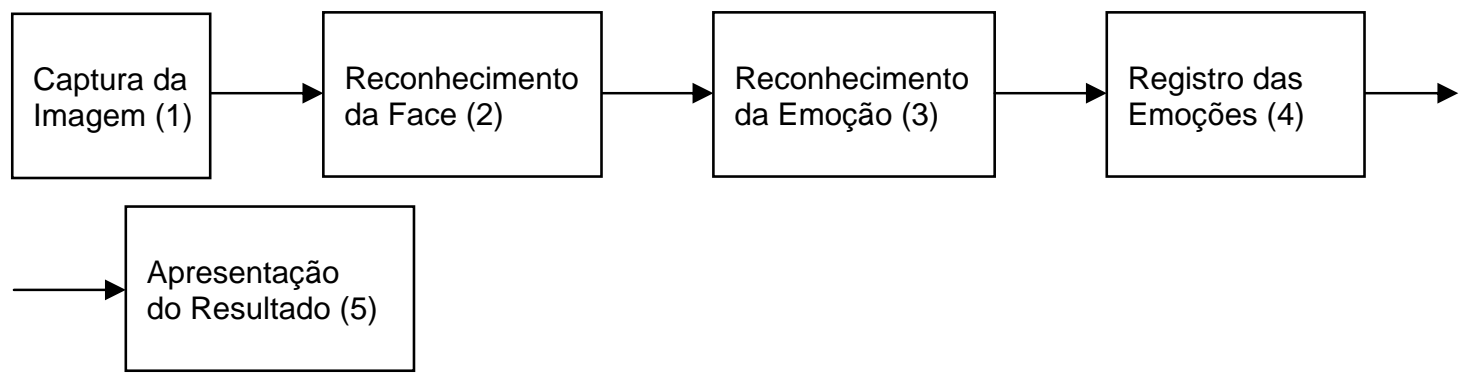

Os sistemas de captura de imagem (1) e reconhecimento de face (2) pertencem à visão computacional. Existem exemplos de algoritmos e programas bem desenvolvidos e que podem ser utilizados (Ver Popa, Cohn, e Wang como exemplos, bem como o vídeo exemplo). O reconhecimento da emoção (3) necessita de um banco de imagens a ser associado. Pode-se 
naturalmente usar um banco de imagens padrão como Cohn-Kanade ou MMI, mas de acordo com a probabilidade de o público brasileiro responder com diferentes particularidades emocionais ao longo do tempo no quesito satisfação, é importante desenvolver um banco de expressões brasileiro validado para investigar essa possibilidade. Portanto o reconhecimento da emoção divide-se em dois subsistemas interligados: o reconhecimento pelo algoritmo propriamente dito e a base de dados comparativa, que no caso precisa ser criada. O registro das emoções (4), entretanto, é completamente novo. Não verificamos registro de análise emocional no tempo e sua relação com a satisfação nos casos apresentados de análise de expressões emocionais em outros sistemas. Este trabalho deve ser motivo de estudos futuros. A apresentação dos resultados (5) pode ser desenvolvida através de estudos de interface e $\mathrm{HCl}$ bem fundamentados e conhecidos e que não necessitam serem descritos aqui.

\section{Desenvolvimento.}

Dois sistemas, portanto precisam ser desenvolvidos. O primeiro sistema não é novo. Pode ser fundamentado nos estudos de Ekman e Tracy para a criação de bancos de expressões emocionais validados. O segundo é. Este sistema depende da linha de cada emoção no tempo e de um resultado baseado nessas linhas que é, contudo, subjetivo. Provavelmente o resultado da satisfação emocional depende de um conjunto de emoções atuando no tempo, e é possível que essa variação emocional seja dependente de cultura. Tal subsistema não existe até onde sabemos. Estes são tópicos importantes e que deverão ser tratados no futuro, a saber: a construção da relação entre satisfação e emoções pontuais e a própria construção do registro. Para o caso atual no Brasil, é preciso, em primeiro lugar desenvolver o método e configurá-lo na forma de um sistema aplicável. O primeiro passo é o desenvolvimento do banco de imagens.

\section{Referências}

Cohn, Jeffrey F.; Zlochower, Adena J.; Lien, J.; Kanade, Takeo. 1999. Automated face analysis by feature point tracking has high concurrent validity with manual FACS coding. In: Psychophysiology, Cambridge University Press: 35-43.

Damásio, Antônio R. 1996. O erro de Descartes emoção razão e o cérebro humano. Tradução de Dora Vicente e Georgina Segurado. 1.ed. São Paulo: Companhia das Letras.

Damásio, Antônio R. 2004. Em Busca de Espinoza - prazer e dor na ciência dos sentimentos. 1.ed. São Paulo: Companhia das Letras.

Ekman, P. 1999. Basic Emotions. In: T. Dalgleish and M. Power (Eds.). Handbook of Cognition and Emotion. Sussex, U.K.: John Wiley \& Sons, Ltd.

Ekman, P.; Campos, J.; Davidson R.J.; De Waals. 2003. F. Darwin, Deception, and Facial Expression. In: Emotions Inside Out. Volume 1000. New York: Annals of the New York Academy of Sciences: 205-221.

Ekman, P.; Friesen, W.; Hager, J. C. 2002. Facial Action Coding System. UT, USA: A Human Face.

Ekman, P. 1999. Facial Expressions. In Dalgleish, T., \& Power, M. (Eds.). Handbook of Cognition and Emotion. New York: JohnWiley \& Sons Ltd.

Ekman, P. 1993. Facial Expression and Emotion. American Psychologist. v.48, n.4: 384-392.

Kashimura, K.; Kurosu, M. 1994. The Structure Of The Screen Design And The Cognitive Process. In: Japanese Psychological Association, 58., Disponível em: <http://www.sigchi.org/chi95/proceedings/shortppr/mk_bdy.htm>. Acesso em: 15 Mai. 2008.

Love, Terence. 2002. Constructing a coherent cross-disciplinary body of theory about designing and designs: some philosophical issues. Design Studies. v.23: 345-361.

Meira, L.; Peres, F. 2004. A dialogue-based approach for evaluating educational sotware. Interacting with computers. v.16: 615-633.

Micro Expression Training Tool. Disponível em <http://www.mettoline.com>. Acesso em 30 jan. 2009.

Moraes, A. (Org). 2002. Design e avaliação de interface: ergodesign e interação humanocomputador. 1. ed. Rio De Janeiro: iUsEr. 
Norman, Donald A. 2005. Emotional Design: Why We Love (Or Hate) Everyday Things. [1th] Ed. New York: Basic Books.

Petterson, Rune. 2007. Research in Information Design. In: Congresso Internacional de Design da Informação, 3., Curitiba. Anais...Curitiba: SBDI.

Popa, Mirela.; Rothkrantz, Leon.;Wiggers, Pascal. 2010. Products Appreciation by Facial Expressions Analysis. In: CompSysTech'10.

Sociedade Brasileira de Design da Informação. Disponível em : <http://www.sbdi.org.br/>. Acesso em: 15 mai. 2008.

Tractinsky, Noam. 1997. Aesthetics and Apparent Usability: Empirically Assessing Cultural and Methodological Issues. Disponível em: http://www.sigchi.org/chi97/proceedings/paper/ht.htm.

Tracy, Jessica L.; Robins, Richard W.; Schriber, Roberta A. 2009. Development of a FACS-Verified Set of Basic and Self-Conscious Emotion Expressions. In: American Psychological Association, Vol.9, N. 4: 554-559.

Wang, Jun.; Yin, Lijun. 2007. Static topographic modeling for facial expression. Recognition and analysis. In: Comput. Vis. Image Understand.

\section{Sobre o autor}

Cláudio Santos de Almeida, mestrando em design pela Universidade Federal de Pernambuco, tem realizado atividades de pesquisa e desenvolvimento de sistemas de ambientes 3D interativos, avaliação de software, usabilidade e design emocional.

e-mail: claudio.santos.almeida@gmail.com. 